\title{
RADIAL NERVE PALSY ASSOCIATED WITH LOCALIZED SUBCUTANEOUS FAT NECROSIS IN THE NEWBORN
}

\author{
BY \\ REGINALD LIGHTWOOD \\ From the Paediatric Unit, St. Mary's Hospital Medical School, University of London
}

Many writers use the terms "sclerema neonatorum' and 'adiponecrosis subcutanea neonatorum' in a synonymous sense, and this has caused some confusion, although the real and clinically useful distinction drawn by Gray (1926) between the generalized and serious variety of subcutaneous fat necrosis, and the localized and benign type has become widely accepted. In this paper two cases of the localized type, formerly referred to as 'pseudosclerema', are recorded, because in both of them there was a patch of sclerema situated over the radial nerve trunk above the elbow, and paralytic wrist-drop resulted. Probably this association has been seen before, but it must be uncommon, and I have not read of it in the literature. Clinically it is not without importance since, were it not for the sclerema patch, a lesion of the brachial plexus might be suspected.

The localized type of subcutaneous fat necrosis is a self-limited condition occurring in the neonatal period and is characterized by circumscribed patches of induration (pseudosclerema), which are usually situated in the buttocks, the cheeks, and over the deltoid or scapular areas, situations in which local pressure may occur during delivery so that ischaemia is assumed to be an important causative factor. But this explanation is less easily accepted when it is recalled that the condition has been described in infants born by Caesarian section and Gray (1926) even recorded its presence antenatally. The low proportion of olein which is known to be a feature of the subcutaneous fat of newborn infants is thought to predispose to fat necrosis, and a reduced body temperature may play a secondary part in some instances. Holzel (1951) has recently emphasized the frequency of anoxaemia derived from asphyxia as a likely predisposing factor. The aetiology of sclerema has been discussed by Bojlén and Petri (1936), Zeek and Madden (1946), and by Holzel (1951).

\section{Case Reports}

Case 1. An illegitimate boy was born on December 26,1949 , in Paddington Hospital following a labour which lasted 100 hours and terminated in normal delivery.

There had been no opportunities for antenatal examination.

After birth there were no signs of asphyxia in the infant, but a mucus extractor was used to clear the airpassages which were somewhat bubbly. No other type of resuscitative measure was required, and the only injection was of 'synkavit ', $100 \mathrm{mg}$. of which were given into the outer aspect of the right buttock. There is no need to give details of the baby's general progress since this was normal. The birth weight was $8 \mathrm{lb} .4 \mathrm{oz}$. and the length 22 in. The following interesting abnormalities were noted.

On the outer side of the left upper arm, slightly above the elbow and overlying the radial nerve, there was an area of skin about the size of an almond which was dusky red and indurated. This was apparently present 'at the time of birth', and was thought by the obstetrician to have been caused by intra-uterine pressure. Its presence attracted special attention to the baby, and examination 48 hours after delivery revealed a flaccid paralysis of the left forearm and a drop-wrist. This paralysis was treated by dorsiflexion of the wrist. The wrist-drop persisted until the end of January, 1950, when both it and the patch of induration were disappearing.

The following is the result of a neurological examination made by Dr. C. C. Edwards on January 25:-

'There is a severe degree of wrist-drop on the left side with gross weakness of the extensors of the wrist, fingers, and thumb, but no detectable weakness of any other muscle group, particularly in the hand and above the level of the elbow. There seems to be an area of relative insensitivity to pin-prick over the base of the left thumb. I do not think it is a brachial plexus or root lesion, but believe the infant has a radial nerve palsy. The hard indurated mobile swelling on the outer aspect of the arm above the elbow does not seem to be fixed to the deep tissues, but it overlies a part of the radial nerve which is comparatively superficial, and I imagine this may be relevant to the palsy.'

On February 3 the indurated area of skin and subcutaneous tissue above the left elbow was excised for examination. The histological report stated that the section showed necrosis of fat tissue, and a granulomatous reaction with numerous foreign-body giant cells. By February 15, the indurated area had disappeared, the hand was held in a normal position and moved freely. The baby made good progress and a complete recovery. The mother, on the other hand, had a severe puerperal illness; she developed thrombosis in the deep veins of the 
legs and at the end of the second week a pulmonary embolus, but eventually she made a complete recovery.

The indurated lesion on this baby's left arm was exactly similar to the indurations sometimes developing soon after birth and called pseudosclerema. These patches of 'pseudosclerema' consist of 'traumatic fat necrosis', and are often attributed to compression of subcutaneous tissue overlying certain bony prominences during delivery. The histology corresponded with this condition, and its resolution was similar but the position above the elbow overlying the radial nerve is a site where pressure is not likely to occur during delivery. There is, however, the possibility that during the second stage of labour, which in this case was prolonged, the left arm of the foetus might have been constricted just above the elbow causing a localized patch of sclerema. It is reasonable to conjecture that the radial nerve might have been damaged by compression at the same time, or involved secondarily by the fat necrosis and subsequent tissue reaction; the former is the more probable because the weakness was maximal when first discovered and then lessened.

Case 2. This girl was born on January 6, 1951, at the same hospital as Case 1. The mother was healthy; it was her second pregnancy, and she was at full term. Presentation was a vertex (L.O.A.). The head was delivered easily, with no perineal tear, and the shoulders were born easily about two minutes after the head, the arms folded on the baby's chest. There were no signs of asphyxia. The midwife did not suspect any undue pressure on the arms by pelvic brim, outlet or perineum. When she bathed the baby she did not observe any abnormality. No injections were required.

Three days after birth, on January 9, 1951, 60 hours after delivery, left wrist-drop was noted. The baby could move the arm but could not extend the wrist which hung down when the forearm was held in a prone position. On the same arm I looked for, and found, an indurated red patch one inch above the elbow on the course of the radial nerve trunk. This had the characteristics of a patch of sclerema, and measured $\frac{3}{8}$ in. by $\frac{1}{2}$ in. It involved both skin and subcutaneous tissues, and its centre was slightly depressed. The wrist-drop was over-corrected with a light splint.

One week later the sclerema patch, though still palpable was fading in colour, and the wrist-drop was rapidly improving. On January 23, when the infant was 17 days old, the nodule of sclerema could still be felt above the elbow. In a neurological report, Dr. C. C. Edwards stated that there was 'no abnormality of the nervous system except a residual weakness of the dorsiflexors of left wrist and fingers.'

A final examination was made on March 6 when the baby was 2 months old. The sclerema patch had shrunk to the size of a pea and there was no residual weakness of the left wrist or fingers.

This is another instance in which a patch of sclerema appeared over the course of the radial nerve above the elbow, and again it was associated with radial palsy. A biopsy was not performed. In this patient the onset was probably a little later after birth, and the condition was less severe, for the sclerema patch was smaller, the palsy was not complete, and its recovery was much quicker. It is not possible to say whether the palsy developed before the patch or vice versa, but the weakness was maximal at its onset, as it was in the first case.

\section{Discussion and Summary}

The presence of wrist-drop demands a search for its cause; if it is unilateral there will be a local one. Two cases of this nature in newborn infants are described. The wrist-drop was associated with a small patch of sclerema forming over the supracondylar portion of the radial nerve just above the elbow. This showed the topography of the condition, and enabled all suspicion of brachial plexus injury to be excluded. The association is rare and the prognosis apparently excellent.

Here we see a variant of the usual clinical picture in localized sclerema, and this description is submitted in honour of Sir Robert Hutchison who, by his example, taught his pupils the satisfaction of making an exact clinical observation, particularly when a simple deduction could be drawn from it.

My thanks are due to Dr. M. Bodian, Dr. C. C. Edwards, and also to the staff of the obstetric department of Paddington Hospital.

\section{REFERENCES}

Bojlén, K., and Petri, S. (1936). Acta paediatr., Stockh., $19,123$.

Gray, A. M. H. (1926). Arch. Derm. Syph., Chicago, 14,635 .

Holzel, A. (1951). Archives of Disease in Childhood, 26, 89.

Zeek, P., and Madden, E. M. (1946). Arch. Path., Chicago, 41, 166. 